\title{
PERIODIC SOLUTIONS OF SECOND ORDER HAMILTONIAN SYSTEMS
}

\author{
JAUME LLIBRE $^{1}$ AND AMAR MAKHLOUF ${ }^{2}$
}

\begin{abstract}
We provide sufficient conditions for the existence of periodic solutions of the second order Hamiltonian system$$
-x^{\prime \prime}-\lambda x=\varepsilon V_{x}^{\prime}(t, x),
$$

where $\varepsilon$ is a small parameter, $x \in \mathbb{R}$ and $V(t, x)$ is $2 \pi$-periodic in t. Moreover we provide two applications.
\end{abstract}

\section{INTRODUCTION AND STATEMENT OF THE MAIN RESULTS}

The existence of periodic solutions of the second order Hamiltonian system

$$
-x^{\prime \prime}-\lambda x=V_{x}^{\prime}(t, x),
$$

where $x \in \mathbf{R}^{m}$ and $\mathrm{V}$ is $2 \pi$-periodic in t, has been extensively studied in the last three decades by the variational and others methods see $[1-3,5-15,17-21]$ and the references therein. Here, using the averaging theory, we study the periodic solutions of the second order Hamiltonian system

$$
-x^{\prime \prime}-\lambda x=\varepsilon V_{x}^{\prime}(t, x),
$$

where $\varepsilon$ is a small parameter, $x \in \mathbb{R}$ and $\mathrm{V}(\mathrm{t}, \mathrm{x})$ is $2 \pi$-periodic in $\mathrm{t}$.

To obtain analytically periodic solutions is in general a very difficult work, usually impossible. The averaging theory reduces this difficult problem for the differential equation (1) to find the zeros of a nonlinear function. It is known that in general the averaging theory for finding periodic solutions does not provide all the periodic solutions of the system. For more information about the averaging theory see section 2 and the references quoted there.

We define the functions

$$
\begin{aligned}
& f_{1}\left(x_{0}, y_{0}\right)=\frac{1}{2 \pi r} \int_{0}^{2 \pi s} \sin \left(\frac{r t}{s}\right) V_{x}^{\prime}(t, a(t)) d t \\
& f_{2}\left(x_{0}, y_{0}\right)=-\frac{1}{2 \pi s} \int_{0}^{2 \pi s} \cos \left(\frac{r t}{s}\right) V_{x}^{\prime}(t, a(t)) d t
\end{aligned}
$$

where

$$
a(t)=\frac{1}{r}\left[r x_{0} \cos \left(\frac{r t}{s}\right)+s y_{0} \sin \left(\frac{r t}{s}\right)\right] .
$$

Our main result is the following one.

2010 Mathematics Subject Classification. 37G15, 37C80, 37C30.

Key words and phrases. periodic solution, second order Hamiltonian system, averaging theory. 
Theorem 1. Let $\lambda=r^{2} / s^{2}$ with $r$ and $s$ coprime positive integers. For every $\left(x_{0}^{*}, y_{0}^{*}\right)$ solution of the system

$$
f_{1}\left(x_{0}, y_{0}\right)=0, \quad f_{2}\left(x_{0}, y_{0}\right)=0,
$$

satisfying

$$
\operatorname{det}\left(\left.\frac{\partial\left(f_{1}, f_{2}\right)}{\partial\left(x_{0}, y_{0}\right)}\right|_{\left(x_{0}, y_{0}\right)=\left(x_{0}^{*}, y_{0}^{*}\right)}\right) \neq 0,
$$

the differential equation (1) has a $2 \pi s$-periodic solution $x(t, \varepsilon)$ such that when $\varepsilon \rightarrow 0$ it tends to the periodic solution

$$
x(t)=\frac{x_{0}^{*} \sqrt{\lambda} \cos (\sqrt{\lambda} t)+y_{0}^{*} \sin (\sqrt{\lambda} t)}{\sqrt{\lambda}}
$$

with period $2 \pi s$ of the differential equation $\ddot{x}+\lambda x=0$.

Theorem 1 is proved in section 3. Its proof is based in the averaging theory for computing periodic solutions, see section 2. For others applications of the averaging theory to the study periodic solutions, see [19, 20].

Applications of Theorem 1 are the following.

Proposition 2. Consider the differential equation (1) with $\lambda=4, V(t, x)=$ $\sin ^{2} t\left(a+b x+c x^{2}+d x^{3}\right)$ and $d \neq 0$. Let $A=4 c^{2}-9 b d$ and $B=-3 b d-4 c^{2}$.

(a) If $A \leq 0$ and $B \leq 0$, then the averaging theory does not provide any periodic solution.

(b) If $A>0, B \leq 0$ and $A \pm 4 c \sqrt{A} \neq 0$, then the averaging theory provides two periodic solutions $x_{A}^{ \pm}(t, \varepsilon)$ of the differential equation (1) such that when $\varepsilon \rightarrow 0$ they tend to the periodic solutions $2(2 c \mp \sqrt{A}) \cos (2 t) /(9 d)$ of $\ddot{x}+4 x=0$.

(c) If $A \leq 0$ and $B>0$, then the averaging theory provides two periodic solutions $x_{B}^{ \pm}(t, \varepsilon)$ of the differential equation(1) such that when $\varepsilon \rightarrow 0$ they tend to the periodic solutions $(4 c \cos (2 t) \pm 2 \sqrt{B} \sin (2 t)) /(3 d)$ of $\ddot{x}+4 x=0$.

(d) If $A>0, B>0$ and $A \pm 4 c \sqrt{A} \neq 0$, then the averaging theory provides the four periodic solutions $x_{A}^{ \pm}(t, \varepsilon)$ and $x_{B}^{ \pm}(t, \varepsilon)$ defined in the previous statements.

See figure 1 where are described the regions $(A, B)$ with the corresponding number of periodic orbits.

Proposition 3. For any positive integer $k$ there exists $\varepsilon_{k}>0$ such that for $\varepsilon \in$ $\left(-\varepsilon_{k}, 0\right) \cup\left(0, \varepsilon_{k}\right)$ the differential equation (1) with $\lambda=1$ and $V(t, x)=\cos ^{2} t \cos x$ has at least $k$ periodic solutions of period $2 \pi$.

Propositions 2 and 3 are proved in section 4.

\section{BASIC RESUltS ON AVERAGING THEORY}

In this section we present the basic results from the averaging theory that we shall need for proving the main results of this paper.

We consider the problem of the bifurcation of $T$-periodic solutions from differential systems of the form

$$
\mathbf{x}^{\prime}=F_{0}(t, \mathbf{x})+\varepsilon F_{1}(t, \mathbf{x})+\varepsilon^{2} F_{2}(t, \mathbf{x}, \varepsilon),
$$




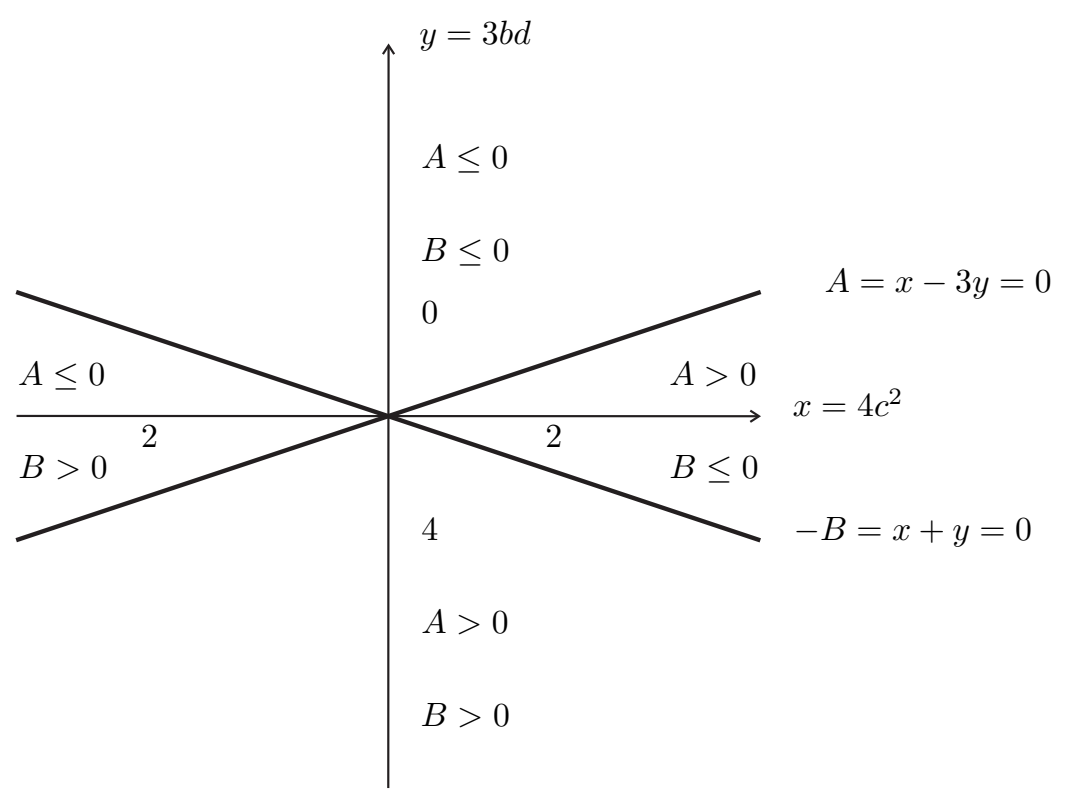

FiguRE 1. The number of periodic orbits in the different regions $(A, B)$.

with $\varepsilon=0$ to $\varepsilon \neq 0$ sufficiently small. Here the functions $F_{0}, F_{1}: \mathbb{R} \times \Omega \rightarrow \mathbb{R}^{n}$ and $F_{2}: \mathbb{R} \times \Omega \times\left(-\varepsilon_{0}, \varepsilon_{0}\right) \rightarrow \mathbb{R}^{n}$ are $\mathcal{C}^{2}$ functions, $T$-periodic in the first variable, and $\Omega$ is an open subset of $\mathbb{R}^{n}$. The main assumption is that the unperturbed system

$$
\mathbf{x}^{\prime}=F_{0}(t, \mathbf{x})
$$

has a submanifold of dimension $n$ of periodic solutions. A solution of this problem is given using the averaging theory.

Let $\mathbf{x}(t, \mathbf{z}, \varepsilon)$ be the solution of the system (5) such that $\mathbf{x}(0, \mathbf{z}, \varepsilon)=\mathbf{z}$. We write the linearization of the unperturbed system along the periodic solution $\mathbf{x}(t, \mathbf{z}, 0)$ as

$$
\mathbf{y}^{\prime}=D_{\mathbf{x}} F_{0}(t, \mathbf{x}(t, \mathbf{z}, 0)) \mathbf{y},
$$

where $\mathbf{y}$ is an $n \times n$ matrix. In what follows we denote by $M_{\mathbf{z}}(t)$ some fundamental matrix of the linear differential system (6).

We assume that there exists an open set $V$ with $\mathrm{Cl}(V) \subset \Omega$ such that for each $\mathbf{z} \in \mathrm{Cl}(V), \mathbf{x}(t, \mathbf{z}, 0)$ is $T$-periodic. The set $\mathrm{Cl}(V)$ is isochronous for the system (4); i.e. it is a set formed only by periodic orbits, all of them having the same period. Then, an answer to the problem of the bifurcation of $T$-periodic solutions from the periodic solutions $\mathbf{x}(t, \mathbf{z}, 0)$ contained in $\mathrm{Cl}(V)$ is given in the following result.

Theorem 4 (Perturbations of an isochronous set). We assume that there exists an open and bounded set $V$ with $\mathrm{Cl}(V) \subset \Omega$ such that for each $\mathbf{z} \in \mathrm{Cl}(V)$, the solution $\mathbf{x}(t, \mathbf{z}, 0)$ is $T$-periodic, then we consider the function $\mathcal{F}: \mathrm{Cl}(V) \rightarrow \mathbb{R}^{n}$

$$
\mathcal{F}(\mathbf{z})=\int_{0}^{T} M_{\mathbf{z}}^{-1}(t) F_{1}(t, \mathbf{x}(t, \mathbf{z}, 0)) d t .
$$


If there exists $\alpha \in V$ with $\mathcal{F}(\alpha)=0$ and $\operatorname{det}((d \mathcal{F} / d \mathbf{z})(\alpha)) \neq 0$, then there exists a T-periodic solution $\mathbf{x}(t, \varepsilon)$ of system (4) such that when $\varepsilon \rightarrow 0$ we have that $\mathbf{x}(0, \varepsilon) \rightarrow \alpha$.

Theorem 4 goes back to Malkin [16] and Roseau [18], for a shorter and easier proof see [4].

\section{Proof of Theorem 1}

If $y=\dot{x}$, then the second-order non-autonomous differential equation (1) can be written as the following first-order differential system in $\mathbb{R}^{2}$

$$
\begin{aligned}
& \dot{x}=y, \\
& \dot{y}=-\lambda x-\varepsilon V_{x}^{\prime}(t, x) .
\end{aligned}
$$

For $\varepsilon=0$ it follows that $(x, y)=(0,0)$ is the unique singular point of system $(8)$. The eigenvalues of the linearized system at this singular point are pure imaginary $\pm i \sqrt{\lambda}$. The solution $(x(t), y(t))$ of the unperturbed system (i.e. system (8) with $\varepsilon=0)$ such that $(x(0), y(0))=\left(x_{0}, y_{0}\right)$ is

$$
\begin{aligned}
& x(t)=\frac{x_{0} \sqrt{\lambda} \cos (\sqrt{\lambda} t)+y_{0} \sin (\sqrt{\lambda} t)}{\sqrt{\lambda}}, \\
& y(t)=y_{0} \cos (\sqrt{\lambda} t)-x_{0} \sqrt{\lambda} \sin (\sqrt{\lambda} t) .
\end{aligned}
$$

Note that all these periodic orbits have period $2 \pi / \sqrt{\lambda}$. We need that $\sqrt{\lambda}$ be rational, $\sqrt{\lambda}=r / s$ with $\mathrm{r}$ and $\mathrm{s}$ coprime positive integer.

Using the notation introduced in section 2 , we have that $\mathbf{x}=(x, y), \mathbf{z}=\left(x_{0}, y_{0}\right)$, $F_{0}(\mathbf{x}, t)=(y,-\lambda x), F_{1}(\mathbf{x}, t)=\left(0,-V_{x}^{\prime}(t, x)\right)$ and $F_{2}(\mathbf{x}, t, \varepsilon)=(0,0)$. The fundamental matrix solution $M_{\mathbf{z}}(t)$ is independent of $\mathbf{z}$ and we shall denote it by $M(t)$. An easy computation shows that

$$
M(t)=\left(\begin{array}{cc}
\cos (\sqrt{\lambda} t) & \frac{\sin (\sqrt{\lambda} t)}{\sqrt{\lambda}} \\
-\sqrt{\lambda} \sin (\sqrt{\lambda} t) & \cos (\sqrt{\lambda} t)
\end{array}\right),
$$

and

$$
M^{-1}(t)=\left(\begin{array}{cc}
\cos (\sqrt{\lambda} t) & -\frac{\sin (\sqrt{\lambda} t)}{\sqrt{\lambda}} \\
\sqrt{\lambda} \sin (\sqrt{\lambda} t) & \cos (\sqrt{\lambda} t)
\end{array}\right) .
$$

According to Theorem 4 we study the zeros $\mathbf{z}=\left(x_{0}, y_{0}\right)$ of the 2 components of the function $\mathcal{F}(\mathbf{z})$ given in $(7)$. More precisely we have $\mathcal{F}(\mathbf{z})=\left(f_{1}(\mathbf{z}), f_{2}(\mathbf{z})\right)$, with

where

$$
\begin{aligned}
& f_{1}(\mathbf{z})=\frac{1}{2 \pi r} \int_{0}^{2 \pi s} \sin \left(\frac{r t}{s}\right) V_{x}^{\prime}(t, a(t)) d t \\
& f_{2}(\mathbf{z})=-\frac{1}{2 \pi s} \int_{0}^{2 \pi s} \cos \left(\frac{r t}{s}\right) V_{x}^{\prime}(t, a(t)) d t
\end{aligned}
$$

$$
a(t)=\frac{1}{r}\left[r x_{0} \cos \left(\frac{r t}{s}\right)+s y_{0} \sin \left(\frac{r t}{s}\right)\right] .
$$




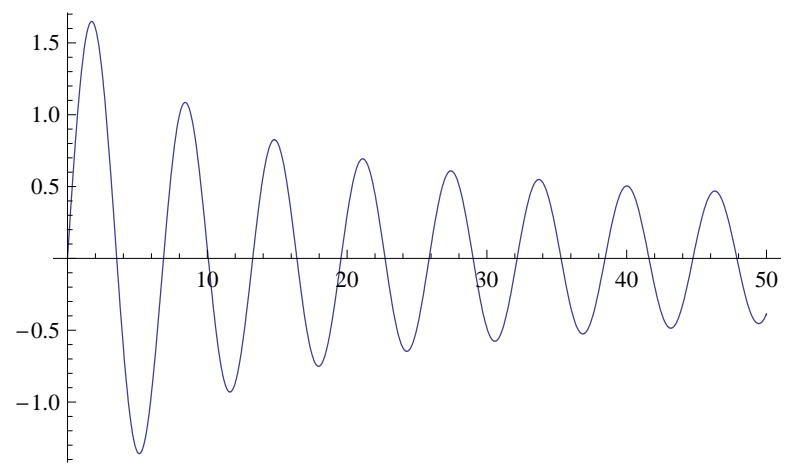

FiguRE 2. The zeros of the function $3 J_{1}(R)-J_{3}(R)$.

Now the rest of the proof of Theorem 1 follows directly from the statement of Theorem 4.

\section{Proof of propositions}

Proof of Proposition 2. We must apply Theorem 1 with $V(t, x)=\sin ^{2} t(a+b x+$ $\left.c x^{2}+d x^{3}\right)$. After computing the functions $f_{1}$ and $f_{2}$ of Theorem 1 we obtain

$$
\begin{aligned}
& f_{1}\left(x_{0}, y_{0}\right)=\frac{1}{32}\left(4 c-3 d x_{0}\right) y_{0}, \\
& f_{2}\left(x_{0}, y_{0}\right)=\frac{1}{64}\left(16 b-32 c x_{0}+3 d\left(12 x_{0}^{2}+y_{0}^{2}\right)\right) .
\end{aligned}
$$

Then system $f_{1}=f_{2}=0$ has four solutions $\left(x_{0}^{*}, y_{0}^{*}\right)$ given by

$$
\left(\frac{2(2 c \mp \sqrt{A})}{9 d}, 0\right),\left(\frac{4 c}{3 d}, \pm \frac{4 \sqrt{B}}{3 d}\right) .
$$

Since the Jacobian

$$
\operatorname{det}\left(\left.\frac{\partial\left(f_{1}, f_{2}\right)}{\partial\left(x_{0}, y_{0}\right)}\right|_{\left(x_{0}, y_{0}\right)=\left(x_{0}^{*}, y_{0}^{*}\right)}\right)
$$

for these four solutions is respectively

$$
\frac{1}{192}(A \pm 4 c \sqrt{A}),-\frac{1}{64} B
$$

We obtain using Theorem 1 the periodic solutions given in the statement of the proposition.

Proof of Proposition 3. Instead of working with the variable $\left(x_{0}, y_{0}\right)$ we shall work with the variable $(R, \theta)$ with $R>0$ and $\theta \in \mathbb{S}^{1}$, given by

$$
x_{0}=R \cos \theta, \quad y_{0}=R \sin \theta .
$$




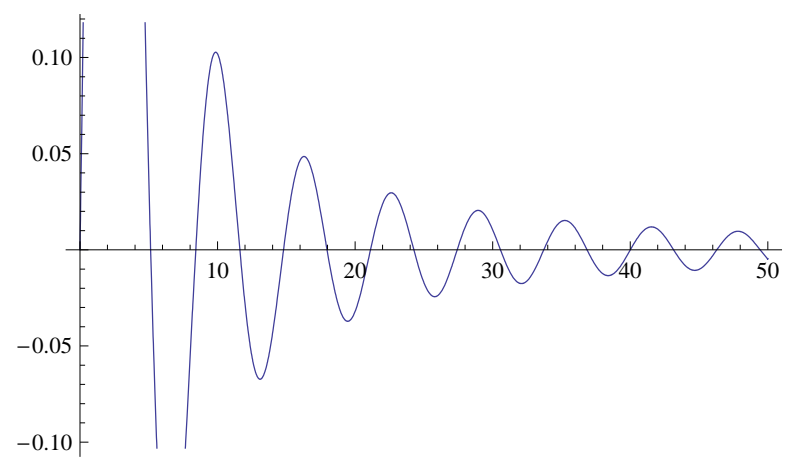

FiguRE 3. The zeros of the function $J_{1}(R)+J_{3}(R)$.

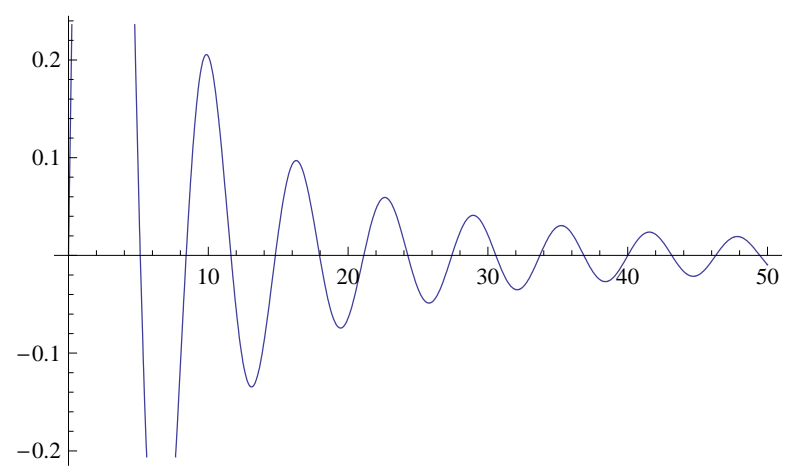

Figure 4. The zeros of the function $8 J_{2}(R) / R$.

Then from Theorem 1, we have

$$
\begin{aligned}
& f_{1}(R, \theta)=-\frac{1}{2 \pi} \int_{0}^{2 \pi} \cos ^{2} t \sin t \sin (R \cos (t-\theta)) d t \\
& f_{2}(R, \theta)=\frac{1}{2 \pi} \int_{0}^{2 \pi} \cos ^{3} t \sin (R \cos (t-\theta)) d t .
\end{aligned}
$$

Computing these integrals we obtain that

$$
\begin{aligned}
& f_{1}(R, \theta)=\frac{1}{4} \sin \theta\left[J_{3}(R)(1+2 \cos (2 \theta))-J_{1}(R)\right], \\
& f_{2}(R, \theta)=\frac{1}{4} \cos \theta\left[J_{3}(R)(1-2 \cos (2 \theta))+3 J_{1}(R)\right],
\end{aligned}
$$

where $J_{n}(z)$ is the $n-t h$ Bessel function of the first kind. The solutions $\left(R^{*}, \theta^{*}\right)$ of system $f_{1}(R, \theta)=f_{2}(R, \theta)=0$ are

(i) $\theta^{*}=0, \pi$ and $R^{*}$ a zero of $3 J_{1}(R)-J_{3}(R)$ (see Fig. 2 );

(ii) $\theta^{*}= \pm \frac{\pi}{2}$ and $R^{*}$ a zero of $J_{1}(R)+J_{3}(R)$ (see Fig. 3); and 
(iii) $\cos \left(2 \theta^{*}\right)=\frac{3 J_{1}(R)+J_{3}(R)}{2 J_{3}(R)}$ and $R^{*}$ a zero of $\frac{8 J_{2}(R)}{R}$ (see Fig. 4 ).

In fact for every zero $R^{*}$ of $(i)$ the two values $\theta^{*}=0, \pi$ provide two different conditions of the same possible periodic orbit. The same occur for (ii) and (iii). In short, for every zero $R^{*}$ of the functions of $(i),(i i)$, and (iii) and for $\varepsilon \neq 0$ sufficiently small the differential equation (1) has a periodic solution if the corresponding Jacobian is nonzero. Using the properties of the Bessel functions it is not difficult to show that the Jacobian

$$
\operatorname{det}\left(\left.\frac{\partial\left(f_{1}, f_{2}\right)}{\partial(R, \theta)}\right|_{(R, \theta)=\left(R^{*}, \theta^{*}\right)}\right)
$$

is different from zero for $\left(R^{*}, \theta^{*}\right)$ satisfying $(i)$, and is zero for the ones satisfying (ii) and $(i i i)$. But since the function $3 J_{1}(R)-J_{3}(R)$ has infinitely many zeros, the statement of the corollary follows.

\section{ACKNOWLEDGEMENTS}

The first author is partially supported by a MICINN/FEDER grant MTM200803437, by an AGAUR grant number 2009SGR-410, and by ICREA Academia.

\section{REFERENCES}

[1] A. Avila and P. Felmer, Periodic and subharmonic solutions for a class of Hamiltonian systems, Dynamics Systems Appl. 3 (1994) 519-536.

[2] A. Bahri and H. Berestycki, Existence of forced oscillations for some nonlinear differential equations, Comm. Pure Appl. Math. 37 (1984) 403-442.

[3] A.K. Ben Naoum, C. Troestler and M. Willem, Existence and multiple results for homogeneous second order differential equations, J. Differential Equations 112 (1994) 239-249.

[4] A. Buica, J. P. Françoise and J. Llibre, Periodic solutions of nonlinear periodic differential systems with a small parameter, Comm. Pure Appl. Anal. 6 (2006), 103-111.

[5] G. Chen and Y. Long, Periodic solutions of superquadratic Hamiltonian systems of second order with mean value zero, Chinese Ann. Math. 19 (1998) 525-532 (In Chinese).

[6] I. Ekeland, Convexity Methods in Hamiltonian Mechanics, Springer, Berlin, New York, 1990.

[7] P. Felmer and E. Silva, Subharmonic solutions near an equilibrium for some second order Hamiltonian systems, Proc. Roy. Soc. Edinburgh 123A (1993) 818-834.

[8] M. Girardi and M. Matzeu, Existence and multiplicity results for periodic solutions of superquadratic Hamiltonian systems where the potential changes sign, Nonlinear Differential Equations Appl. 2 (1995) 35-61.

[9] M. Girardi and M. Matzeu, On periodic solutions of the system $\ddot{x}(t)+b(t)\left(V_{1}^{\prime}(x(t))+\right.$ $\left.V_{2}^{\prime}(x(t))\right)=0$ where $b($.$) changes sign and V_{1}, V_{2}$ have different superquadratic growths, in: Proceedings Local and Variational Methods in Hamiltonian systems, Word Scientific, Singapore, 1995, pp. 65-76.

[10] M.Y. Jiang, Subharmonic solutions of second order subquadratic Hamiltonian system with potential changing sign, J. Math. Anal. Appl. 244 (2000) 291-303.

[11] M.Y. Jiang, Periodic solutions of second order superquadratic Hamiltonian sytems with potentail changing sign (I), J. Differential Equations 219 (2005) 323-341.

[12] M.Y. Jiang, Periodic solutions of second order superquadratic Hamiltonian sytems with potentail changing sign (II), J. Differential Equations 219 (2005) 342-362.

[13] L. Lassoued, Solutions périodiques d'un système différentiel non linéaire du second ordre avec changement de signe, Anal. Mat. Pura Appl. 156 (1990) 76-111.

[14] L. Lassoued, Periodic solutions of a second order superquadratic system with change of sign of the potential, J. Differential Equations 93 (1991) 1-18.

[15] Y. Long, Multiple solutions of perturbed superquadratic second order hamiltonian systems, Trans. Amer. Math. Soc. 311 (1989) 749-780. 
[16] I. G. Malkin, Some Problems of the theory of nonlinear oscillations, Gosudarstv. Izdat. TehnTeor. Lit. Moscow, 1956 (in Russian).

[17] P.H. Rabinowitz, Minimax Methods in Critical Point Theory with Applications to Differential Equations, CBMS, Regional Conference Series in Mathematics, Vol. 65, American Mathematical Society, Providence, RI, 1986.

[18] M. Roseau, Vibrations non linéaires et théorie de la stabilité, Springer Tracts in Natural Philosophy, Vol. 8, Springer, New York, 1985.

[19] J. A. Sanders and F. Verhulst, Averaging Methods in Nonlinear Dynamical Systems, Applied Mathematical Sciences 59, Springer, 1985.

[20] F. Verhulst, Nonlinear Differential Equations and Dynamical Systems, Universitext, Springer, New York, 1996.

[21] W. Zou and S. Li, Infinity many periodic solutions of Hamiltonian systems, J. Differential Equations 160 (2002) 223-253.

1 Departament de Matematiques, Universitat Autònoma de Barcelona, 08193 Bellaterra, Barcelona, Catalonia, Spain

E-mail address: jllibre@mat.uab.cat

2 Department of Mathematics, University of Annaba, Elhadjar, 23 Annaba, Algeria

E-mail address: makhloufamar@yahoo.fr 